\title{
On-line parameter estimation of a magnetic bearing
}

\author{
Romain Delpoux and Thierry Floquet
}

\begin{abstract}
This article presents a parameter estimation algorithm for a magnetic bearing. Such process are inherently unstable systems with strongly nonlinear dynamics. Here, a simplified model of the magnetic bearing is developed. This enables to obtain a linear expression with respect to the unknown parameters. These parameters are measurable with difficulties, and may slightly vary over time. The expression of the estimates is written as a function of integrals of the inputs and outputs of the system. The simulations and the experiments show a fast and robust on-line identification.
\end{abstract}

Index Terms-Parameter estimation, Laplace transform, Magnetic bearing.

\section{INTRODUCTION}

Magnetic bearings can be used for machine tool spindles to produce circular and non-circular holes with high precision. Specially in high speed applications, magnetic bearings won importance. Because they have a contact free suspension, they provide advantages compared to conventional bearings such as no lubrification and no frictions. This improves reliability and performances. There is a real industrial demand for such process which allow non-circular motion of the order of 50 micro-meters. The required precision is really important: Path tracking error must be less than 1 micrometer on circular paths and 3 micro-meters on non-circular ones with a rotation speed up to $1000 \mathrm{rpm}$.

The magnetic shaft used in our laboratory consists of one electromagnetic radial bearing and two axial bearings. It is similar to the one present in [4]. It is an inherently unstable system where the dynamics are strongly nonlinear. We refer to the work [3], [4], [8], [9], [10] concerning the control of magnetic shaft.

This paper is concerned with the estimation of parameters of the radial bearing using an algebraic approach based on the work of Fliess and Sira-Ramirez [6], [7]. The estimation procedure, given by exact formulas, leads to a non asymptotic convergence. In this approach, it is possible to express the desired parameters as a function of integrals of the measured output and the inputs of the system. This method has already been applied to parameter estimation [11], [13], to abrupt change detection and delays [1], [2], [5], [16] as well as to numerical differentiation [12], [15].

This article is divided into three parts. Section II presents the radial magnetic bearing and introduces problems encountered

This work was supported by Nord-Pas de Calais Regional Council and FEDER through the Contrat de Projets Etat Region (CPER) 2007-2013.

R. Delpoux and T. Floquet are with the Laboratoire d'Automatique, Génie Informatique et Signal (CNRS FRE 3303), École Centrale de Lille, 59651 Villeneuve d'Ascq cedex, France and Team Non-A, INRIA Lille-Nord Europe, France. romain. delpouxdec-lille.fr and thierry.floqueteec-lille.fr when identifying parameters. In Section III, expressions for the on-line identification of the desired parameters are developed. Here is treated the on-line estimation of parameters depending on the radial bearing geometry and the materials. The last Section is devoted to simulation and experimental results.

\section{Problem Statement}

In this article is presented an approach to estimate parameters of the radial bearing (the axial bearing case will be considered in future work). The parameters that are to be estimated here depend on the geometry and the material of the bearing. The estimation of these parameters is important because such parameters are difficult to calculate and may slightly vary over time.

The rotor is levitating using a three-phase electromagnetic radial bearing, arranged like three coupled "horseshoe magnets" around the rotor (Fig. 1). The three generated current provide three independent control inputs. The mathematical model of the bearing is based on the assumption of a rigid body and leads to decoupled equations for forces. The dynamics equations, under simplifying assumptions, are written as follows:

$$
\begin{aligned}
m \ddot{Y} & =F_{y} \\
m \ddot{Z} & =F_{z}
\end{aligned}
$$

where $\mathrm{Y}$ and $\mathrm{Z}$ represent the coordinates of the center of mass of the rotor in a Cartesian frame (with axes $y$ et $z$ ) which is fixed in the space, at a point being considered as the center of the device. The forces $F_{y}$ and $F_{z}$ represent the resulting forces applied in directions $y$ et $z$, respectively. The rotor has a mass $m$. The resultant forces in the plan $(y-z)$ are given by the superposition of the forces generated by the

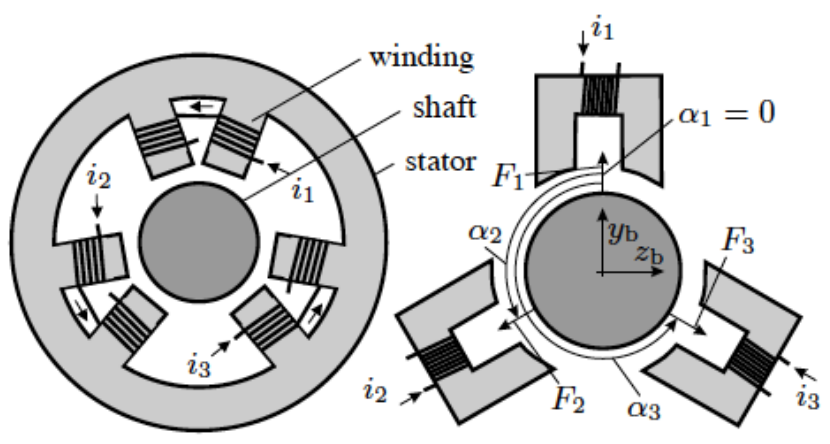

Fig. 1. Sketch of the three-phase radial bearing 
magnets:

$$
\left(\begin{array}{c}
F_{y} \\
F_{z}
\end{array}\right)=\left(\begin{array}{ccc}
\sin \alpha_{1} & \sin \alpha_{2} & \sin \alpha_{3} \\
\cos \alpha_{1} & \cos \alpha_{2} & \cos \alpha_{3}
\end{array}\right)\left(\begin{array}{c}
F_{1} \\
F_{2} \\
F_{3}
\end{array}\right)
$$

The angles that appear in (3) are presented Fig. 1. Individually, the magnetic forces can be modeled by $(k \in\{1,2,3\})$

$$
F_{k}=\lambda_{k} \frac{i_{k}^{2}}{\left(s-\left(\begin{array}{c}
\sin \alpha_{k} \\
\cos \alpha_{k}
\end{array}\right)^{T}\left(\begin{array}{c}
Y_{b} \\
Z_{b}
\end{array}\right)\right)^{2}}
$$

where $Y_{b}$ and $Z_{b}$ are the positions in the bearing plan. $s$ is the nominal air gap and $\lambda_{k}$ are parameters depending on the geometry and the materials of the bearing. They will be estimated on-line.

Calculating control currents: The reference currents are obtained from the desired forces. In order to simplify the notations, we assume that the bearing is symmetric, i.e. $\alpha_{1}=$ $\pi, \alpha_{2}=-\frac{\pi}{3}$ and $\alpha_{3}=\frac{\pi}{3}$. In each of the models detailed above, we obtain a couple of independent forces for each bearing plan. Define $F_{1}$ such that:

$$
F_{1}= \begin{cases}F_{0} & \text { if } \quad F_{z} \geq \frac{\left|F_{y}\right|}{\sqrt{3}} \\ F_{0}-\frac{\left|F_{y}\right|}{\sqrt{3}}+F_{z} & \text { else }\end{cases}
$$

With $F_{0} \geq 0$ arbitrarily chosen, the two remaining forces are obtained from the model (3):

$$
\begin{aligned}
& F_{2}=F_{1}+\frac{F_{y}}{\sqrt{3}}+F_{z} \\
& F_{3}=F_{1}-\frac{F_{y}}{\sqrt{3}}+F_{z}
\end{aligned}
$$

Then the currents are calculated from the desired magnetic forces using relation (4).

The above equations reflect the dynamics of the unperturbed model. Model simplifications in (1)-(2) and modeling errors (e.g. due to incorrect relationship between the input currents $i_{*}$ and the corresponding forces $F_{*}$ ) are assumed to have the same effect than perturbations.

The rotor rotation is also a perturbation source with harmonic components. When the rotor moves along an ellipsoid trajectory, the positions in the directions $y$ et $z$ are sinusoidal functions. A significant error in the relation between the currents and the forces can also change sinusoidally.

Therefore, the perturbations must be rejected in the $\lambda_{i}$ estimation algorithm (or must be estimated in order to be compensated in the control).

The perturbed model can be written as:

$$
\begin{aligned}
m \ddot{Y} & =F_{y}+p_{y}(t) \\
m \ddot{Z} & =F_{z}+p_{z}(t)
\end{aligned}
$$

where $p_{*}(t)$ represent the perturbations on each axis. Using equation (6), (7), (3) and (4), we obtain a relationship of the form:

$$
\begin{aligned}
\ddot{Y} & =\sum_{k=1}^{3} \lambda_{k} u_{y, k}+\frac{p_{y}(t)}{m} \\
\ddot{Z} & =\sum_{k=1}^{3} \lambda_{k} u_{z, k}+\frac{p_{z}(t)}{m}
\end{aligned}
$$

$$
\text { with } u_{y, k}=\frac{\sin \left(\alpha_{k}\right) i_{k}^{2}}{m\left(s-\left(\begin{array}{c}
\sin \alpha_{k} \\
\cos \alpha_{k}
\end{array}\right)^{T}\left(\begin{array}{c}
Y_{b} \\
Z_{b}
\end{array}\right)\right)^{2}}
$$

These two equations depend on the measured inputs and the accelerations in the plan. The parameters $\lambda_{k}$ are the parameters to be estimated from measurements $Y$ and $Z$ and despite the perturbations. They may be difficult to compute. The identification of magnetic bearings is usually slow to be implemented and is often made off-line. In the next Section, we will develop expressions for the identification leading to an on-line, fast and robust estimation of these parameters.

\section{Algebraic APPROACH}

\section{A. Estimation of $\lambda_{k}$ parameters}

It will be shown in this Section, that the three parameters $\lambda_{1}, \lambda_{2}$ and $\lambda_{3}$ can be estimated using equation (9). Indeed, using an algebraic approach for this system, it is possible to express the parameters $\lambda_{k}$ as a function of the measured outputs and the inputs only. In order to simplify the computations, it is assumed that the perturbations are constant. We will see in the next paragraph how to deal with non constant perturbations.

Consider the Laplace transform of (9) :

$s^{2} Z(s)-s Z(0)-\dot{Z}(0)=\lambda_{1} U_{Z, 1}(s)+\lambda_{2} U_{Z, 2}(s)+\lambda_{3} U_{Z, 3}(s)+\frac{a}{s}$

and multiply (10) by $s$ :

$$
\begin{aligned}
& s^{3} Z(s)-s^{2} Z(0)-s \dot{Z}(0)= \\
& \lambda_{1} s U_{Z, 1}(s)+\lambda_{2} s U_{Z, 2}(s)+\lambda_{3} s U_{Z, 3}(s)+a
\end{aligned}
$$

Derive three times with respect to $s$ to cancel the initial conditions and the perturbations:

$$
\begin{aligned}
& 6 Z(s)+18 s \frac{d}{d s}(Z(s))+9 s^{2} \frac{d^{2}}{d s^{2}}(Z(s))+s^{3} \frac{d^{3}}{d s^{3}}(Z(s)) \\
& =\lambda_{1}\left(3 \frac{d^{2}}{d s^{2}}\left(U_{Z, 1}(s)\right)+s \frac{d^{3}}{d s^{3}}\left(U_{Z, 1}(s)\right)\right) \\
& +\lambda_{2}\left(3 \frac{d^{2}}{d s^{2}}\left(U_{Z, 2}(s)\right)+s \frac{d^{3}}{d s^{3}}\left(U_{Z, 2}(s)\right)\right) \\
& +\lambda_{3}\left(3 \frac{d^{2}}{d s^{2}}\left(U_{Z, 3}(s)\right)+s \frac{d^{3}}{d s^{3}}\left(U_{Z, 3}(s)\right)\right)
\end{aligned}
$$

We remind that differentiation with respect to $s$ in the operational domain results in a multiplication by $-t$ in the time domain. The multiplication by $s$ in the operational domain leads to the derivation in the time domain. The application of the linear estimator (12) is then not convenient. Derivation amplify the high frequency and then the noise contribution. A simple solution is to make the estimator proper. We simply divide (12) by $s^{4}$ in order to cancel 
the derivation terms and obtain a relationship with integral operators:

$$
\begin{aligned}
& 6 s^{-4} Z(s)+18 s^{-3} \frac{d}{d s}(Z(s))+9 s^{-2} \frac{d^{2}}{d s^{2}}(Z(s))+s^{-1} \frac{d^{3}}{d s^{3}}(Z(s)) \\
& =\lambda_{1}\left(3 s^{-4} \frac{d^{2}}{d s^{2}}\left(U_{Z, 1}(s)\right)+s^{-3} \frac{d^{3}}{d s^{3}}\left(U_{Z, 1}(s)\right)\right) \\
& +\lambda_{2}\left(3 s^{-4} \frac{d^{2}}{d s^{2}}\left(U_{Z, 2}(s)\right)+s^{-3} \frac{d^{3}}{d s^{3}}\left(U_{Z, 2}(s)\right)\right) \\
& +\lambda_{3}\left(3 s^{-4} \frac{d^{2}}{d s^{2}}\left(U_{Z, 3}(s)\right)+s^{-3} \frac{d^{3}}{d s^{3}}\left(U_{Z, 3}(s)\right)\right)
\end{aligned}
$$

To return to the time domain, compute the inverse Laplace transform and apply the following formula:

$\int_{0}^{t} \int_{0}^{t_{k-1}} \ldots \int_{0}^{t_{1}} x(\tau) d \tau d t_{1} \ldots d t_{k-1}=\frac{1}{(k-1) !} \int_{0}^{t}(t-\tau)^{k-1} x(\tau) d \tau$

One gets:

$$
\begin{array}{ll} 
& \int_{0}^{t}\left[\frac{6}{3 !}(t-\tau)^{3}-\frac{18}{2}(t-\tau)^{2} \tau+9(t-\tau) \tau^{2}-\tau^{3}\right] Z(\tau) d \tau \\
= & \lambda_{1}\left(\int_{0}^{t}\left[\frac{3}{3 !}(t-\tau)^{3} \tau^{2}-\frac{1}{2}(t-\tau)^{2} \tau^{3}\right] u_{Z, 1}(\tau) d \tau\right) \\
+ & \lambda_{2}\left(\int_{0}^{t}\left[\frac{3}{3 !}(t-\tau)^{3} \tau^{2}-\frac{1}{2}(t-\tau)^{2} \tau^{3}\right] u_{Z, 2}(\tau) d \tau\right) \\
+ & \lambda_{3}\left(\int_{0}^{t}\left[\frac{3}{3 !}(t-\tau)^{3} \tau^{2}-\frac{1}{2}(t-\tau)^{2} \tau^{3}\right] u_{Z, 3}(\tau) d \tau\right)
\end{array}
$$

The previous equation depends on the integrals of the measured outputs and inputs. However, there is three unknown parameters for one equation. Then, we have to generate two more equations in order to have the same number of equations than unknowns. To obtain a second equation, take equation (11) but differentiating it four times with respect to $s$ and apply the same manipulations as before. We obtain:

$$
\begin{aligned}
\int_{0}^{t}[- & \left.\frac{24}{3 !}(t-\tau)^{3} \tau+\frac{36}{2}(t-\tau)^{2} \tau^{2}-12(t-\tau) \tau^{3}+\tau^{4}\right] Z(\tau) d \tau \\
& =\lambda_{1}\left(\int_{0}^{t}\left[-\frac{4}{3 !}(t-\tau)^{3} \tau^{3}+\frac{1}{2}(t-\tau)^{2} \tau^{4}\right] u_{Z, 1}(\tau) d \tau\right) \\
& +\lambda_{2}\left(\int_{0}^{t}\left[-\frac{4}{3 !}(t-\tau)^{3} \tau^{3}+\frac{1}{2}(t-\tau)^{2} \tau^{4}\right] u_{Z, 2}(\tau) d \tau\right) \\
& +\lambda_{3}\left(\int_{0}^{t}\left[-\frac{4}{3 !}(t-\tau)^{3} \tau^{3}+\frac{1}{2}(t-\tau)^{2} \tau^{4}\right] u_{Z, 3}(\tau) d \tau\right)
\end{aligned}
$$

The last equation is obtained, from equation (11) but differentiating it five times with respect to $s$. One gets:

$$
\begin{aligned}
& \int_{0}^{t}\left[\frac{60}{3 !}(t-\tau)^{3} \tau^{2}-\frac{60}{2}(t-\tau)^{2} \tau^{3}+15(t-\tau) \tau^{4}-\tau^{5}\right] Z(\tau) d \tau \\
& \quad=\lambda_{1}\left(\int_{0}^{t}\left[\frac{5}{3 !}(t-\tau)^{3} \tau^{4}-\frac{1}{2}(t-\tau)^{2} \tau^{5}\right] u_{Z, 1}(\tau) d \tau\right) \\
& \quad+\lambda_{2}\left(\int_{0}^{t}\left[\frac{5}{3 !}(t-\tau)^{3} \tau^{4}-\frac{1}{2}(t-\tau)^{2} \tau^{5}\right] u_{Z, 2}(\tau) d \tau\right) \\
& \quad+\lambda_{3}\left(\int_{0}^{t}\left[\frac{5}{3 !}(t-\tau)^{3} \tau^{4}-\frac{1}{2}(t-\tau)^{2} \tau^{5}\right] u_{Z, 3}(\tau) d \tau\right)
\end{aligned}
$$

Define the parameter vector: $\Lambda=\left[\begin{array}{lll}\lambda_{1} & \lambda_{2} & \lambda_{3}\end{array}\right]^{(17)}$. Equations (15), (16) and (17) allow to obtain the expressions of the estimate of the parameters $\hat{\lambda}_{1}, \hat{\lambda}_{2}$ et $\hat{\lambda}_{3}$ :

$$
D(t) \hat{\Lambda}=N(t)
$$

with the matrices $D(t) \in \mathbb{R}^{3 \times 3}$ et $N(t) \in \mathbb{R}^{3 \times 1}$. If $D(t)$ is invertible, one gets:

$$
\left[\begin{array}{l}
\hat{\lambda}_{1} \\
\hat{\lambda}_{2} \\
\hat{\lambda}_{3}
\end{array}\right]=D(t)^{-1} N(t)
$$

Remark 1: Note that at time $t=0$, the matrices and vectors used to compute the estimations are null. The parameters are then undetermined. Therefore, the formula has to be calculated, not at the time $t=0$, but at a later time, $t=\epsilon$ with $\epsilon>0$ and small.

Thus the parameter vector is estimated as follows :

$$
\hat{\Lambda}= \begin{cases}\text { arbitrary } & \text { for } t \in[0, \epsilon[ \\ D(t)^{-1} N(t) & \text { for } t \in[\epsilon, \infty[\end{cases}
$$

\section{B. Implementation}

In the previous paragraph, it was assumed that the perturbations were constant. Nevertheless, this is physically not realistic. It has been seen in Section II that there are two different perturbation sources.

The first perturbation source, due to simplifications and modeling error, can be considered as a constant if the signals are integrating over a small sliding window. So far, the integrals have been computed over an interval $[0, t]$. However integrating over a short time interval $\left[0, T_{f}\right]\left(T_{f}\right.$ represents the window length), we can consider that the perturbations are constant. Finally we use a change of variable to reduce the estimation interval $\mathcal{I}_{0}^{T_{f}}=\left[0, T_{f}\right]$ to $[0,1] . N(t)$ and $D(t)$ can be rewritten as:

$$
N(t)=\left[\int_{0}^{1} g_{z, i}(\tau) Z(\tau) d \tau\right]_{1 \leq i \leq 3}
$$

and

$$
D(t)=\left[\int_{0}^{1} g_{u_{z, i}}(\tau) U_{Z, j}(\tau) d \tau\right]_{1 \leq i \leq 3,1 \leq j \leq 3}
$$

with

$$
\begin{aligned}
& g_{z, 1}(\tau)=\left[\frac{6}{3 !}(1-\tau)^{3}-\frac{18}{2}(1-\tau)^{2} \tau+9(1-\tau) \tau^{2}-\tau^{3}\right] T_{f}^{3} \\
& g_{z, 2}(\tau)=\left[-\frac{24}{3 !}(1-\tau)^{3} \tau+\frac{36}{2}(1-\tau)^{2} \tau^{2}-12(1-\tau) \tau^{3}+\tau^{4}\right] T_{f}^{4} \\
& g_{z, 3}(\tau)=\left[\frac{60}{3 !}(1-\tau)^{3} \tau^{2}-\frac{60}{2}(1-\tau)^{2} \tau^{3}+15(1-\tau) \tau^{5}-\tau^{5}\right] T_{f}^{5} \\
& g_{u_{z, 1}}(\tau)=\left[\frac{3}{3 !}(1-\tau)^{3} \tau^{2}-\frac{1}{2}(1-\tau)^{2} \tau^{3}\right] T_{f}^{5} \\
& g_{u_{z, 2}}(\tau)=\left[-\frac{4}{3 !}(1-\tau)^{3} \tau^{3}+\frac{1}{2}(1-\tau)^{2} \tau^{4}\right] T_{f}^{6} \\
& g_{u_{z, 3}}(\tau)=\left[\frac{5}{3 !}(1-\tau)^{3} \tau^{4}-\frac{1}{2}(1-\tau)^{2} \tau^{5}\right] T_{f}^{7}
\end{aligned}
$$

Using the trapezoidal method, the integrals are obtained from the output of a classical FIR filter.

To reject the harmonic perturbations with frequencies close to rotational speed, the resulting numerator and denominator of each estimated parameters are filtered using a low-pass filter. The filter used is similar to the one presented in [14] :

$$
\hat{\lambda}_{i, f}(t)=\frac{F(s) n(t)}{F(s) d(t)}
$$

where $F(s)=\frac{\omega_{n}^{2}}{s^{2}+2 \zeta \omega_{n}+\omega_{n}^{2}}$.

Experimental results are shown in the next Section.

\section{RESUlts}

Simulation and experimental results are presented in this section. The numerical simulations are carried out in order to show the performances of the algorithm. Finally, we give the estimation results on the real process.

The parameters are the rotor mass $m=6.7(\mathrm{~kg})$ and the nominal air gap $s=5 \cdot 10^{-4}(\mathrm{~m})$. The control is realized as a cascade, with a current controller in the inner loop and a position controller in the outer loop. The inner loop can be characterised by a control based on the electrical model of the bearing coils. The outer is based on the rigid body 

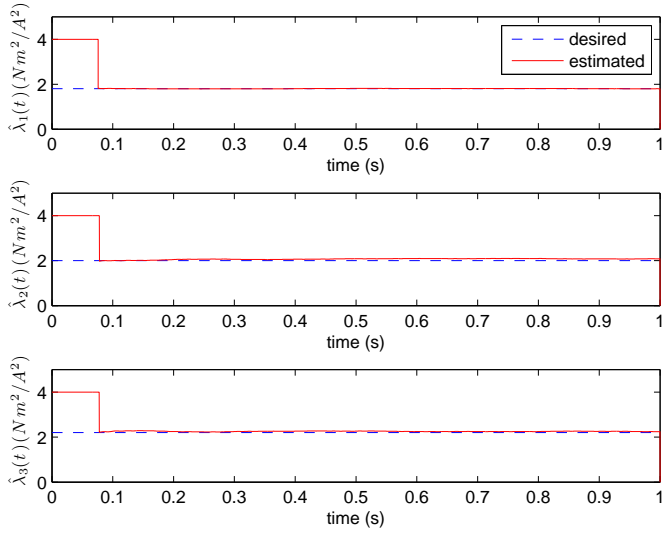

Fig. 2. Parameter estimation without noise

mechanical model (6)-(7). The chosen control design is a flatness based trajectory tracking control, as described in [10]. The reference trajectory is an elliptic one where $Y^{*}=$ $r_{Y} \cos (\omega t)$ and $Z^{*}=r_{Z} \sin (\omega t)$ with $r_{Y}=30.10^{-6}(\mathrm{~m})$ and $r_{Z}=70 \cdot 10^{-6}(\mathrm{~m})$. The shaft is rotating with an angular velocity of about $3000 \mathrm{rpm}$, i.e. $\omega=50 \cdot 2 \pi(\mathrm{rad} / \mathrm{s})$.

The estimator is implemented in a discretized way with a sampling frequency of $1.10^{-4}(\mathrm{~s})$. The choice of the window length is not straightforward. The window has to be sufficiently long to cancel the noise but not too long to keep the assumption on the constant perturbation true. The experience shows that a window $T_{f}=0.02 \mathrm{~s}$ gives good results. The initial value of $\lambda_{k, 0}$ is set arbitrarily. The filter $F(s)$ is chosen with $\zeta=0.707$ and $w_{n}=15 \mathrm{rad} / \mathrm{s}$.

At the beginning of the experiment, the controller uses the initial and arbitrary $\lambda_{k, 0}$ from $t=0$ to $t=t_{e}, t_{e}$ being the convergence time of the algorithm. The estimator, connected in parallel to the system, estimates the parameters $\hat{\lambda}_{k}$ in real time on the interval $\left(\epsilon, t_{e}\right]$. As soon as the estimations have converged to constant values, the initial values $\lambda_{k, 0}$ are replaced by the estimated ones.
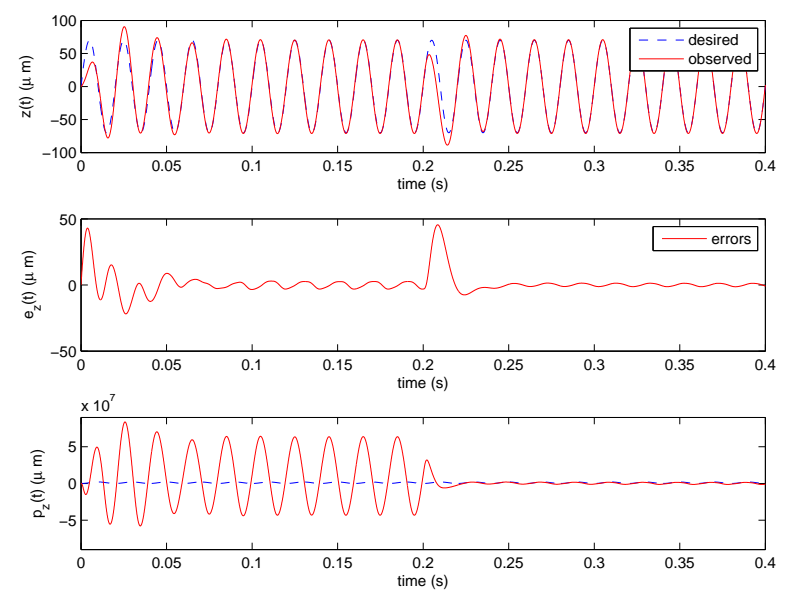

Fig. 3. Trajectory tracking evolution of $\mathrm{z}$ axe

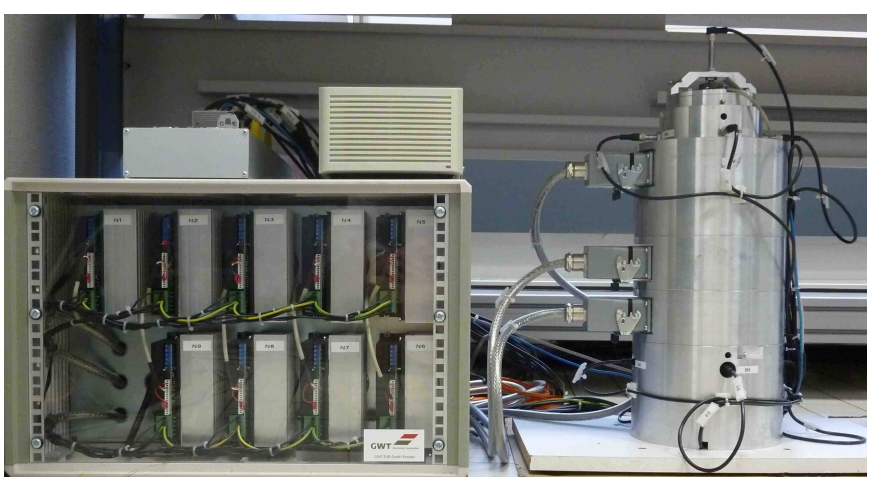

Fig. 4. Magnetic bearing test-bench

\section{A. Simulation Results}

In the simulations, the real values of the parameters $\lambda_{k}$ are chosen as $\lambda_{1}=1.8 .10^{-6}, \lambda_{2}=2.0 .10^{-6}, \lambda_{1}=2.2 .10^{-6}$. The initial value of the estimation is $\lambda_{k, 0}=4 \cdot 0 \cdot 10^{-6}$. To estimate the parameters, we choose sinusoidal perturbations with non zero mean value to be close to the real perturbations.

Fig. 2 presents the parameters estimation. In this figure, it can be seen that the three parameters converge to the desired value in less than 0.1 second with $\hat{\lambda}_{1}=1.786 .10^{-6}$, $\hat{\lambda}_{2}=2.072 .10^{-6}$ and $\hat{\lambda}_{3}=2.199 .10^{-6}$. It represents an error less that $3.5 \%$. Fig. 3 depicts the trajectory tracking evolution of the z-axis only (the y-axis is similar). The estimated parameters are updated after 0.2 seconds. Before identification, the position errors are already small. However, the perturbations estimation is far from the real perturbation. It reflects the modeling errors. After parameters update, the position error is slightly decreased while the perturbation error is considerably decrease. The model is more correct, and the observed perturbation is close to the real one.

\section{B. Experimental Results}

In this Section, the experimental results are presented. The test-bench is shown Fig. 4. The computer hardware on the

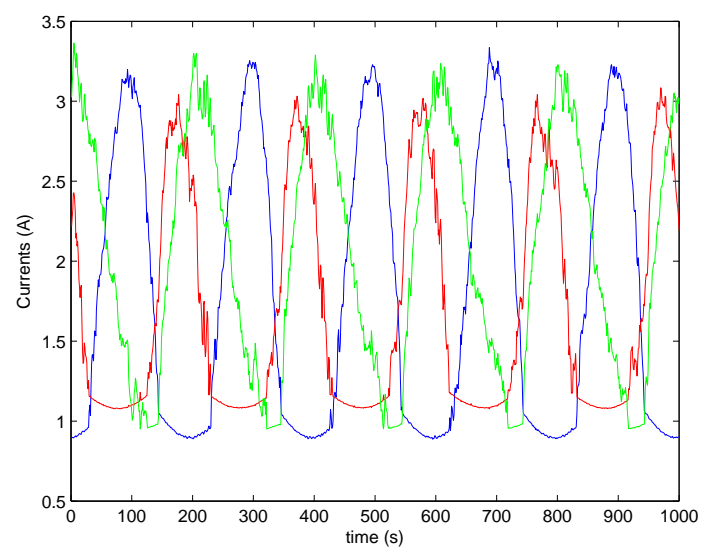

Fig. 5. Control Currents 

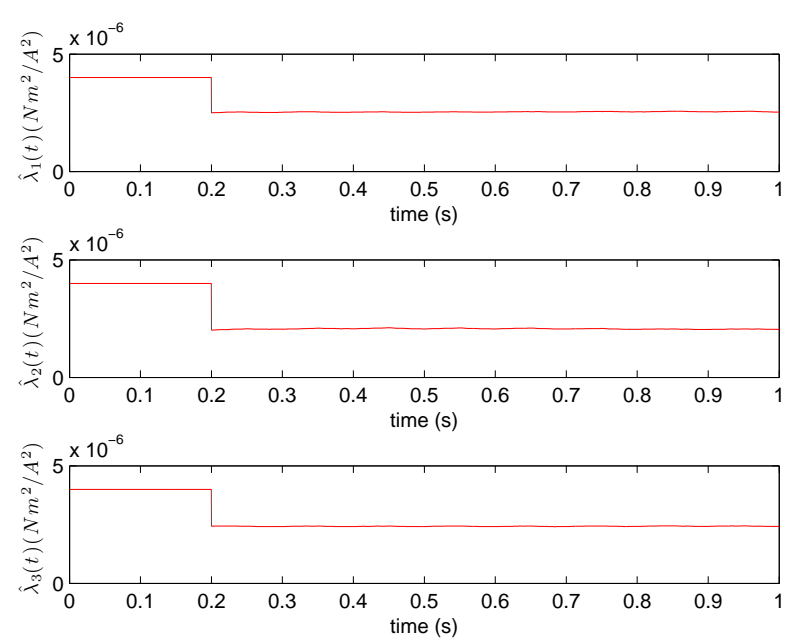

Fig. 6. Experimental estimation of the parameters

test-bench is a dSpace 1103. The control law is implemented in $\mathrm{C}$ and linked to the mechanical unit through Control Desk software. All currents are generated by 3 Dc brush amplifiers that serve as three independent control inputs (Fig. 5).

When the experiment starts, one does not have precise knowledge of $\lambda_{k}$. As in the simulations, the parameters are set arbitrarily equal to $4 \cdot 0 \cdot 10^{-6}\left(\mathrm{Nm}^{2} / \mathrm{A}^{2}\right)$. The estimation algorithm starts at the beginning of the experiment. Fig. 6 shows that after 0.2 seconds, the parameters have converged. Indeed, one obtains three constant values for the parameters $\left(\hat{\lambda}_{1}=2.53 .10^{-6}, \hat{\lambda}_{2}=2.04 .10^{-6}\right.$ and $\left.\hat{\lambda}_{1}=2.42 .10^{-6}\right)$.

In order to check estimation accuracy of the parameters, we observe the trajectories and the perturbations before and after estimation. Fig. 7 and 8 show the evolution of the $y$ and $z$ axes before identification while Fig. 9 and 10 show the evolution after identification. Similarly to the simulation results, the most obvious result is on the perturbations. An estimate of the latter is obtained using the perturbation observer given in [10]. The amplitude of the perturbations is
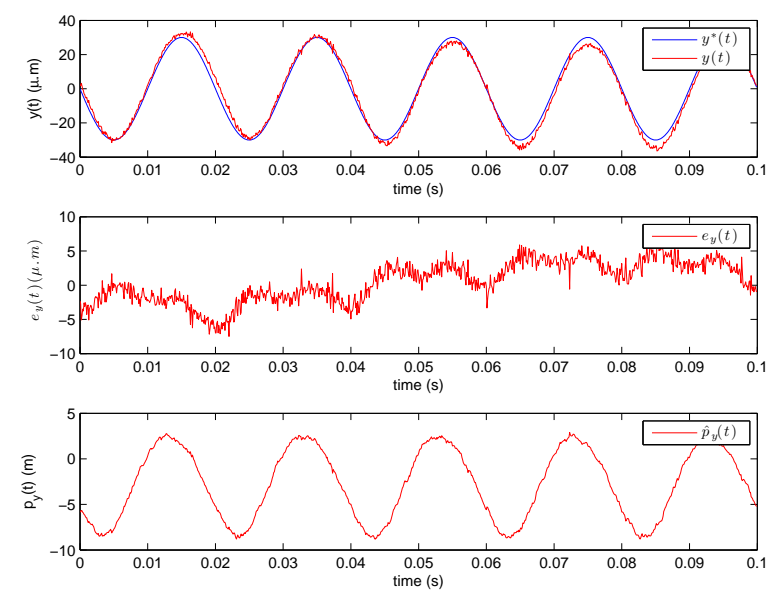

Fig. 7. Trajectory tracking evaluation on the $y$ axe before identification
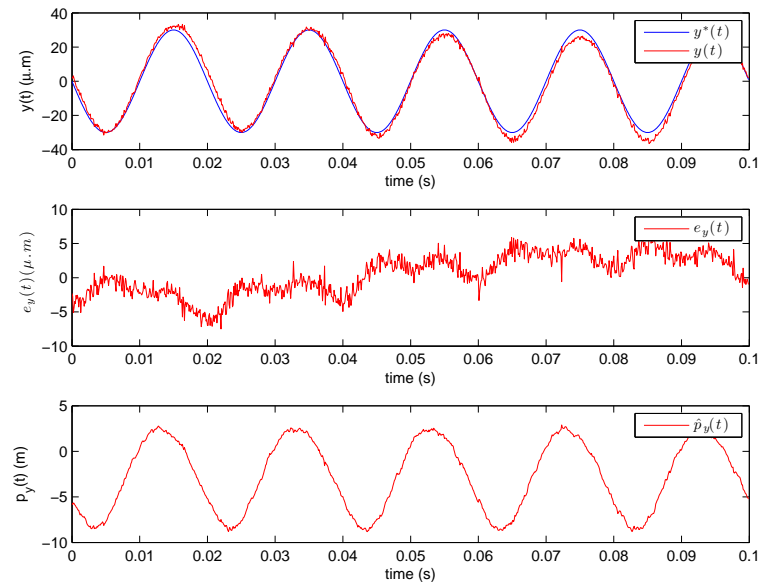

Fig. 8. Trajectory tracking evaluation on the $z$ axe before identification

at least three times smaller after identification than before and leads to a significant reduction of the modeling uncertainties. Concerning the trajectories tracking and their errors, the improvement is less sensitive. Looking at the errors, it can be seen that the positions errors are slightly lower when the parameters are well estimated and more regular. The behavior is not really different, but this is not surprising. Indeed, the designed control law compensate for the perturbations which are themselves estimated by an observer. Estimating the perturbation, the observer takes in consideration the modelling error. Finally the last plots (Fig. 11 and 12) gives an idea of the shape of the hole before and after identification.

\section{CONCLUSIONS AND FUTURE WORKS}

In this article, it has been presented an estimator able to evaluate in a fast way and simultaneously several unknown parameters of a radial magnetic bearing. This system is strongly nonlinear and unstable. The performances of this algorithm show good experimental results. Indeed, the estimation is robust, the estimation error is low in spite of noise
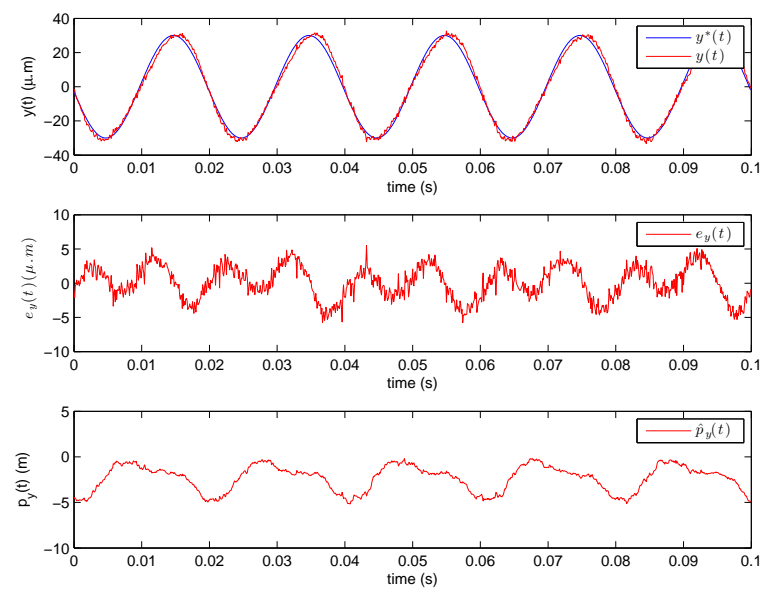

Fig. 9. Trajectory tracking evaluation on the $y$ axe after identification 

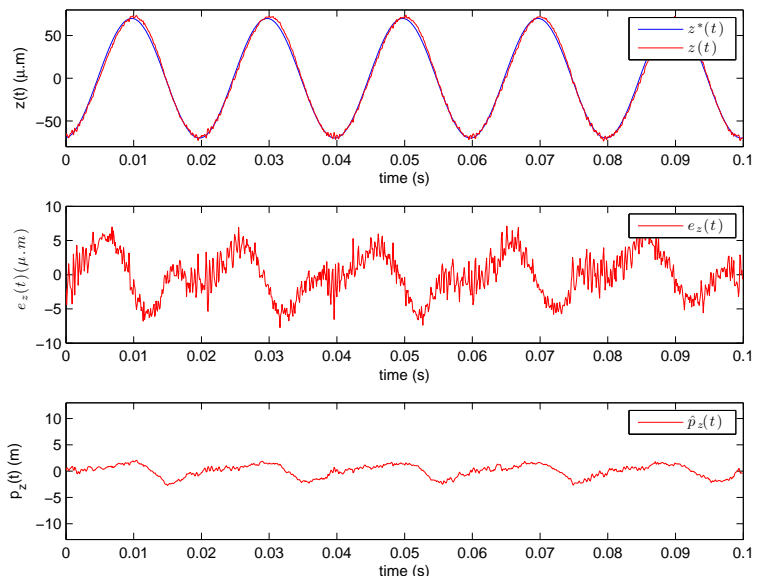

Fig. 10. Trajectory tracking evaluation on the $z$ axe after identification

and perturbations, and also fast with convergence time of 0.2 seconds. Using the estimated parameters, the trajectories are well tracked. Experiments show that the maximum tracking error is around $5 \mu \mathrm{m}$ for a non-circular hole. As improvement, it will by interesting to simultaneously identify the perturbations amplitude. Using better sensors than the ones used could also leads to better tracking performance.

\section{REFERENCES}

[1] L. Belkoura, T. Floquet, K. Ibn Taarit, W. Perruquetti, and Y. Orlov. Estimation problems for a class of impulsive systems. International Journal of Robust and Nonlinear Control, 2010.

[2] L. Belkoura, J.P. Richard, and Fliess M. Parameters estimation of systems with delayed and structured entries. Automatica, 45(5):1117 $-1125,2009$.

[3] Hsu C.-T. and S.-L. Chen. Nonlinear control of a 3-pole active magnetic bearing system. Automatica, 39(2):291-298, Feb 2003.

[4] S. Eckhardt and J. Rudolph. High precision synchronous tool path tracking with an amb machine tool spindle. In Ninth International Symp. on Magnetic Bearings, Lexington, Kentucky, USA, aug 3-6 2004.

[5] M. Fliess, C. Join, and M. Mboup. Algebraic change-point detection. Applicable Algebra in Engineering, Communication and Computing, 21:131-143, 2010.

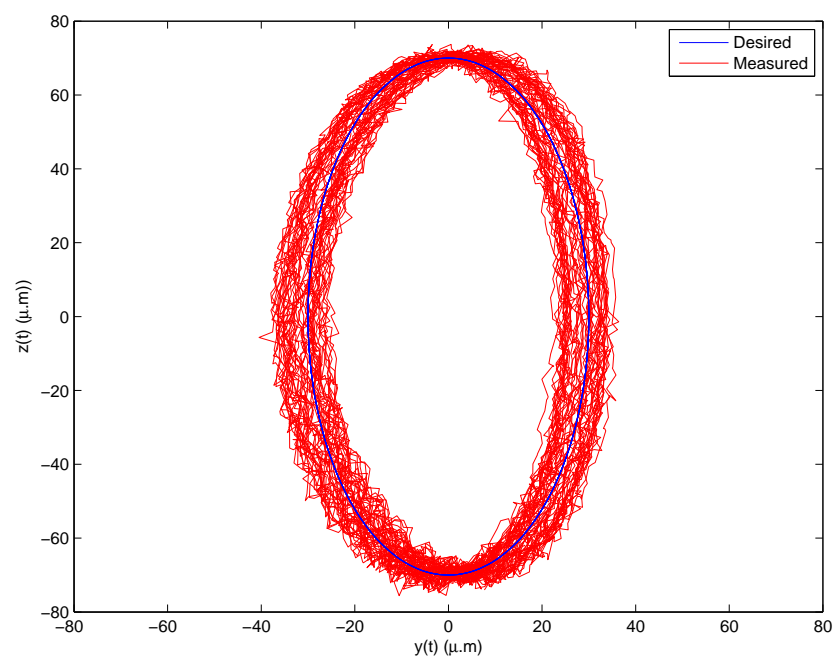

Fig. 11. Position in the $y-z$ plan before identification

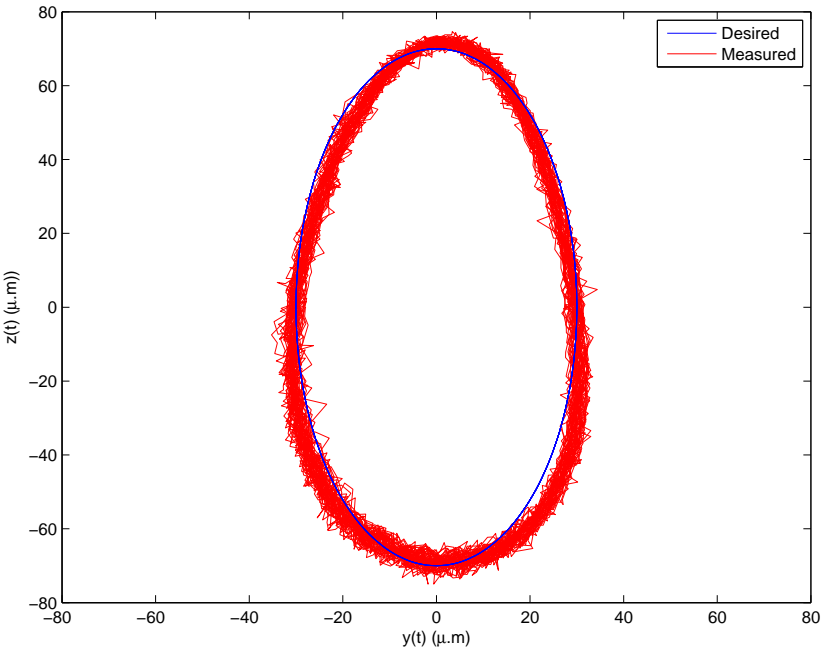

Fig. 12. Position in the $y-z$ plan after identification

[6] M. Fliess and H. Sira-Ramírez. An algebraic framework for linear identification. ESAIM: COCV, 9:151-168, jan 2003.

[7] M. Fliess and H. Sira Ramirez. Closed-loop parametric identification for continuous-time linear systems via new algebraic techniques. In $\mathrm{H}$ Garnier \& L. Wang, editor, Identification of Continuous-time Models from Sampled Data, Advances in Industrial Control, pages 362-391. Springer, 2008.

[8] T. Grochmal and A. Lynch. Control of a self-bearing servomotor. Control Systems Magazine, IEEE, 29(5):74-92, Oct. 2009.

[9] J. Levine, J. Lottin, and J.-C. Ponsart. A nonlinear approach to the control of magnetic bearings. Control Systems Technology, IEEE Transactions on, 4(5):524-544, Sep 1996.

[10] J. v. Lowis and J. Rudolph. Flatness-based trajectory tracking control of a rotationg shaft. In Seventh International Symp. on Magnetic Bearings, Zurich, pages 299-304, August 2000.

[11] M. Mboup. Parameter estimation via differential algebra and operational calculus. Research Report, 2007.

[12] M. Mboup, C. Join, and M. Fliess. Numerical differentiation with annihilators in noisy environment. Numerical Algorithms, 50:439467, 2009.

[13] R. Morales, V. Feliu, and H. Sira-Ramirez. Nonlinear control for magnetic levitation systems based on fast online algebraic identification of the input gain. Control Systems Technology, IEEE Transactions on, PP(99):1 -15, 2010.

[14] J. R. Trapero, H. Sira-Ramirez, and V. Feliu. An algebraic frequency estimator for a biased and noisy sinusoidal signal. Signal Processing, 87(6): 1188 - 1201, 2007.

[15] S. Riachy, Y. Bachalany, M. Mboup, and J.P. Richard. Différentiation numérique multivariable I : estimateurs algébriques et structure. In Sixième Conférence Internationale Francophone d'Automatique Nancy, France, 2-4 juin 2010, Nancy France, 2010.

[16] Z. Tiganj, M. Mboup, C. Pouzat, and L. Belkoura. An algebraic method for eye blink artifacts detection in single channel EEG recordings. In 17th International Conference on Biomagnetism BIOMAG2010, volume 28, pages 175-178, Dubrovnik Croatie, 03 2010. 